\title{
Positron Annihilation Spectroscopy Study of NiMnGa Modulated and Non-Modulated Martensitic Phases
}

\author{
David Merida ${ }^{1, a}$, Jose Angel García ${ }^{1}$, Estibalitz Apiñaniz², \\ Fernando Plazaola ${ }^{2, b}$, Vicente Sanchez-Alarcos ${ }^{3, c}$, \\ Jose Ignacio Pérez-Landazábal ${ }^{3}$ and Vicente Recarte ${ }^{3}$ \\ ${ }^{1}$ Fisika Aplikatua II Saila, Euskal Herriko Unibertsitatea, p.k. 644,48080 Bilbao, Spain \\ ${ }^{2}$ Elektrizitate eta Elektronika Saila, Euskal Herriko Unibertsitatea, p.k. 644,48080 Bilbao, Spain \\ ${ }^{3}$ Departamento de Física, Universidad Pública de Navarra, Campus de Arrosadia, 31006 \\ Pamplona, Spain \\ adavid.merida@ehu.es, bfernando.plazaola@ehu.es, cvicente.sanchez@unavarra.es
}

Keywords: positron annihilation, NiMnGa

\begin{abstract}
We have studied by means of positron lifetime measurements the role that vacancy type defects play in the martensitic transformation in magnetic Ni-Mn-Ga shape memory alloys. The measurements presented in this work have been performed in two ternary alloys transforming to modulate and non-modulated martensitic phases. Positron experiments have been realized at room temperature after subsequent isochronal heating at different temperatures up to a maximum temperature of $600^{\circ} \mathrm{C}$. Positron results show a large variation of the average lifetime value with the isochronal annealing temperature in non-modulated samples. However, the response in the modulated samples is quite different. These results obtained in both samples are discussed in term of different type of positrons trapping defects and their evolution with the annealing temperature. They have also been compared with Differential Scanning Calorimetry experiments performed in the same samples. The work states the clear influence of the vacancy concentration on the martensitic transformations of these alloys.
\end{abstract}

\section{Introduction}

Since giant magnetic-field-induced strain (MFIS) was first reported on Ni-Mn-Ga ferromagnetic shape memory alloys (FSMA) by Ullakko et al. [1], a great amount of work has been performed to understand and improve the system in order to be implemented in practical devices [2-3]. The main drawbacks are the high brittleness and the low values of the martensitic transformation (MT) and Curie temperatures of Ni-Mn-Ga alloys. These limitations have stimulated the research of new ferromagnetic shape memory alloys having better mechanical properties. Indeed, several studies have been performed to improve the mechanical and thermal properties.

Different techniques have been used to analyze diverse properties of Ni-Mn-Ga alloys; however, very little work has been performed to study the role of vacancies in the martensitic transformation. Positron annihilation spectroscopy is a very powerful technique to investigate vacancy-type defects in metals [4]. We have used positron annihilation spectroscopy measurements to study the role of vacancy-type defects in the modulated and non-modulated martensitic transformations of Ni-MnGa polycrystalline alloys. Positron lifetime experiments have been performed at room temperature after subsequent isochronal annealing up to $600^{\circ} \mathrm{C}$. The positron results have been compared with calorimetric experiments performed in the same sample.

\section{Experimental and computational method}

Polycrystalline ingots of $\mathrm{Ni}_{52.6} \mathrm{Mn}_{26.7} \mathrm{Ga}_{20.7}$ and $\mathrm{Ni}_{49.5} \mathrm{Mn}_{28.5} \mathrm{Ga}_{22}$ (at. \%) were prepared from high purity elements by arc melting under protective Ar atmosphere. The ingots were homogenized in vacuum quartz ampoules at $1000^{\circ} \mathrm{C}$ during 24 hours. Small samples for calorimetric measurements 
were obtained from discs previously cut from the center of the ingots by slow speed diamond saw. These discs were used for positron lifetime measurements. Subsequent annealing treatments of 30 minutes at $900^{\circ} \mathrm{C}$ followed by quenching into ice water were performed on the alloys in a vertical induction furnace. The composition of the samples was analyzed before and after thermal treatment by energy-dispersive X-ray spectrometry (EDX) in a JSM-5610LV scanning electron microscope and it was confirmed that no compositional change took place due to thermal treatments. Differential scanning calorimetry (DSC) measurements were carried out in a TA Q100 DSC instrument to study the thermal behavior of the alloys. The samples were polished after quenching in order to ensure a good thermal contact with the equipment. The MT temperatures of the asquenched sample were obtained from a direct measurement up to $400^{\circ} \mathrm{C}$ at a heating/cooling rate of $10 \mathrm{~K} / \mathrm{min}$. In order to observe the evolution of MT temperatures with heat treatment temperature, thermal cycles through the MT after heating up to different temperatures were performed at the same heating/cooling rate. All DSC measurements were performed under nitrogen atmosphere.

For positron lifetime measurements a fast system with a resolution of $235 \mathrm{ps}$ was used and a conventional ${ }^{22} \mathrm{Na}$ source on a kapton foil was employed as a positron source. All lifetime spectra were analyzed after subtracting a constant source contribution: a long source of $1800 \mathrm{ps}$ with an intensity of $1 \%$ and a shorter one of 400 ps with an intensity of $16 \%$. For the isochronal annealing in positron annihilation measurements we used the same heating/cooling rate of $10 \mathrm{~K} / \mathrm{min}$; it means that under such a procedure samples were heated from room temperature to the isochronal annealing temperature and then were cooled back at the same rate to room temperature, where positron lifetime measurements were performed. The points in each positron lifetime curve correspond to the average obtained after 6-10 measurements. The error bars correspond to the maximum deviation of the fitted positron parameters in those measurements.

We have studied two samples; one of them corresponds to a modulated 7M (Pnnm space group) martensitic structure and the other to a non-modulated structure (I4/mmm space group). The composition of the modulated sample is $\mathrm{Ni}_{49.5} \mathrm{Mn}_{28.5} \mathrm{Ga}_{22}(\mathrm{e} / \mathrm{a}=7.605)$ and the composition of the non-modulated one $\mathrm{Ni}_{52.6} \mathrm{Mn}_{26.7} \mathrm{Ga}_{20.7}(\mathrm{c} / \mathrm{a}=7.751)$. We designated the modulated sample as NMG_Mod and the non-modulated one as NMG_NM.

The theoretical calculations have been performed solving first, self-consistently the electron density of the perfect or defected solid, using the tight binding version of the linear muffin-tin orbital method within the atomic-spheres approximation (LMTO-ASA). Then, we have calculated the positron wavefunction, and finally, the positron annihilation rate (the inverse of the positron lifetime) is obtained from the overlap of positron and electron densities as:

$$
\lambda=\pi r_{0}^{2} c \int \mathrm{d} r n_{+}(\mathbf{r}) n_{-}(\mathbf{r}) \gamma(\mathbf{r})
$$

where $r_{0}$ is the classical electron radius, $\mathrm{c}$ is the speed of light in vacuum, $n_{+}(\mathbf{r})$ is the positron density and $\gamma(\mathbf{r})$ is the so-called enhancement factor, which has been taken into account using the generalized gradient approximation. For details about the computational method see reference [5].

The positron lifetime calculations in the cubic structure of $\mathrm{Ni}_{2} \mathrm{MnGa}$, with lattice parameter of $0.58067 \mathrm{~nm}$ [6] at have been performed in a supercell of 16 atoms ( 8 atoms of Ni, 4 atoms of $\mathrm{Mn}$ and 4 atoms of $\mathrm{Ga}$ ).

\section{Results and discussion}

\section{Calorimeter measurements}

Figure 1 shows the results of the DSC measurements carried out up to $400^{\circ} \mathrm{C}$ on the NMG_Mod sample-quenched from $900^{\circ} \mathrm{C}$. An endothermic peak at $20^{\circ} \mathrm{C}$ can be observed corresponding to the reverse MT (martensite-austenite). The change in the baseline at around $80^{\circ} \mathrm{C}$ corresponds to the Curie temperature. It is also worth noting the presence of an exothermic peak at temperatures far above the MT. This peak could be related to processes affecting the MT [7]. The exothermic peak is 
observed only on the first heating up to $400^{\circ} \mathrm{C}$, and it does not appear on subsequent cooling and posterior cycling of the alloy, so the peak must be linked to an irreversible process.

In order to study the origin of the processes related to the exothermic peak, as well as their effect on MT temperatures, consecutive thermal cycles from $150^{\circ} \mathrm{C}$ to $400^{\circ} \mathrm{C}$ inside the exothermic peak temperature range were performed. These intermediate treatments only allow the partial development of the irreversible process.

In order to characterize MT changes we use Mp temperature, the position of the DSC exothermic peak in the MT transformation (austenite-martensite) ) [7], which in the studied samples happens cooling down in the DSC. Figure 2 shows the Mp temperature as a function of isochronal annealing temperature and the exothermic peak of the DSC for the NMG_Mod sample, as-quenched from $900^{\circ} \mathrm{C}$. It can be seen that the beginning of the peak at $200^{\circ} \mathrm{C}$ corresponds to the beginning of the change in the Mp temperature. The maximum of the peak at $300^{\circ} \mathrm{C}$ corresponds to the maximum of the slope of $\mathrm{Mp}$ change rate. The end of the peak at $400^{\circ} \mathrm{C}$ corresponds to the stabilization of the $\mathrm{Mp}$ temperature. In this process the $\mathrm{Mp}$ temperature changes from $12^{\circ} \mathrm{C}$ to $28^{\circ} \mathrm{C}$. $\mathrm{n}$ the other side, a parallel increase of the Curie temperature has been also observed in the same temperature range.

Figure 3 shows the exothermic peak of the DSC and the Mp temperature versus isochronal annealing temperature for the non-modulated NMG_NM sample, as-quenched from $900^{\circ} \mathrm{C}$. In this sample the change of $\mathrm{Mp}$ is delayed in relation with the position of the peak. At $200^{\circ} \mathrm{C}$ the peak starts to grow but there is not any change in $\mathrm{Mp}$ temperature up to $250^{\circ} \mathrm{C}$, at this temperature the peak has arrived to its maximum. The end of the exothermic peak occurs at $300^{\circ} \mathrm{C}$, indeed, the temperature at which the maximum of the slope of $\mathrm{Mp}$ change rate happens. At $350^{\circ} \mathrm{C}$ the $\mathrm{Mp}$ changes ends. The temperature of Curie as well increases with isochronal annealing temperature.

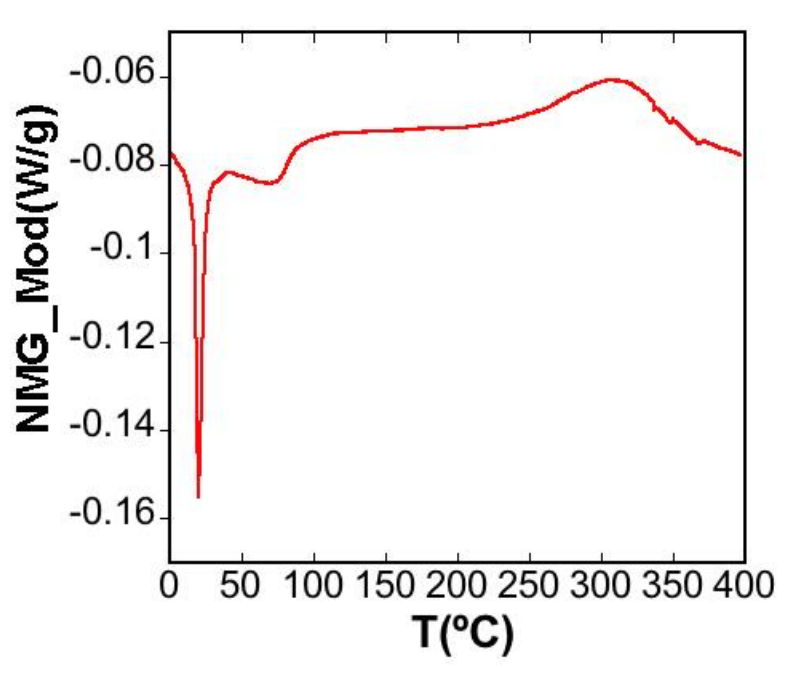

Fig. 1: DSC measurements of NMG_Mod, $\mathrm{Ni}_{49.5} \mathrm{Mn}_{28.5} \mathrm{Ga}_{22}$.

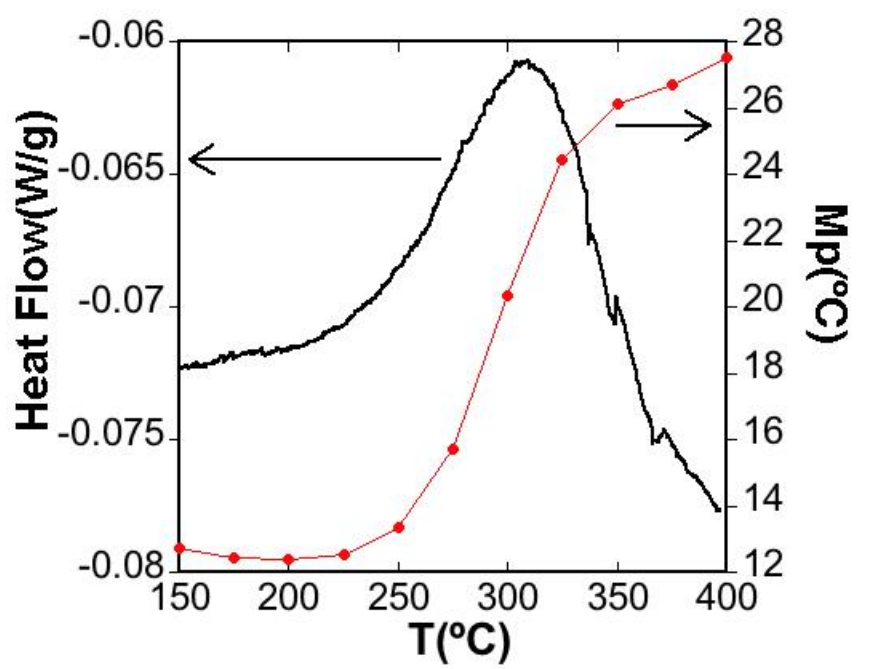

Fig. 2: Exothermic peak of the DSC and martensitic transformation temperature versus isochronal annealing temperature for NMG_Mod.

In the case of the non-modulated NMG_NM sample the presence of small endothermic peaks at temperatures above $200^{\circ} \mathrm{C}$ indicate that the reverse MT has not been fulfilled at this temperature. This stabilization of the martensite points out to defect pinning of the martensite interfaces, which could be a consequence of a high concentration of quenched-in defects in martensite [8].

The calorimetric measurement shows a change in the martensitic transformation temperature during the isochronal annealing process. This can be understood like an ordering process [7]. 


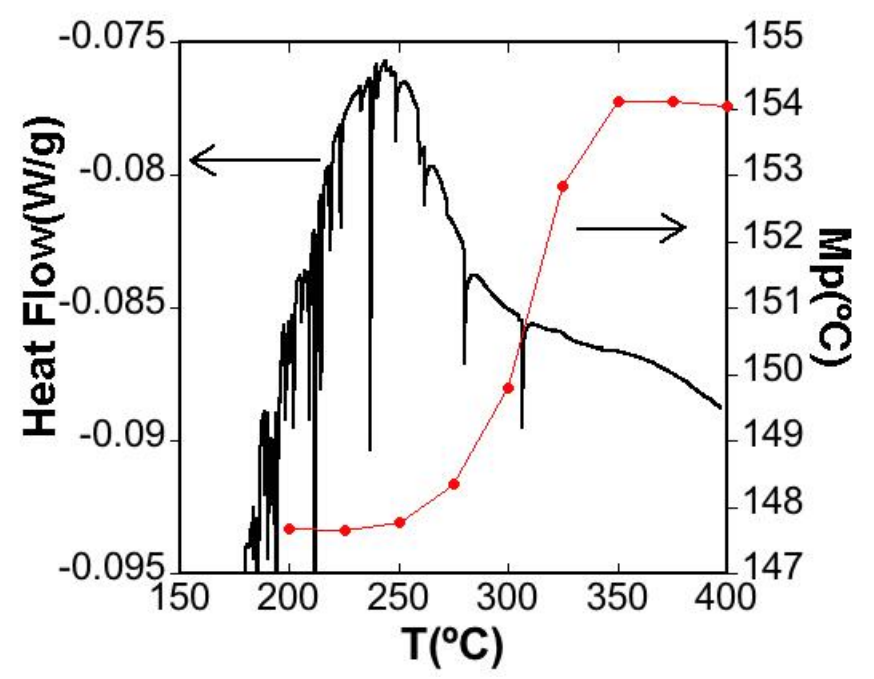

Fig. 3: Exothermic peak of the DSC and the martensitic transformation temperature change versus isochronal annealing temperature of NMG_NM.

\section{Positron lifetimes measurements}

Figure 4 shows the positron average lifetime $\left(\tau_{\mathrm{av}}\right)$ as a function of the isochronal annealing temperature for NMG_Mod and NMG_NM samples. The positron average lifetime of NMG_NM sample shows a strong decrease between $200^{\circ} \mathrm{C}$ and $400^{\circ} \mathrm{C}$ of around $20 \mathrm{ps}$. The temperature range is identical to the one at which the exothermic peak appears. The behavior observed in the NMG_Mod sample is very different. Only a little valley can be seen between $250^{\circ} \mathrm{C}$ and $450^{\circ} \mathrm{C}$, with the overall decrease amounting only to about 3 ps.

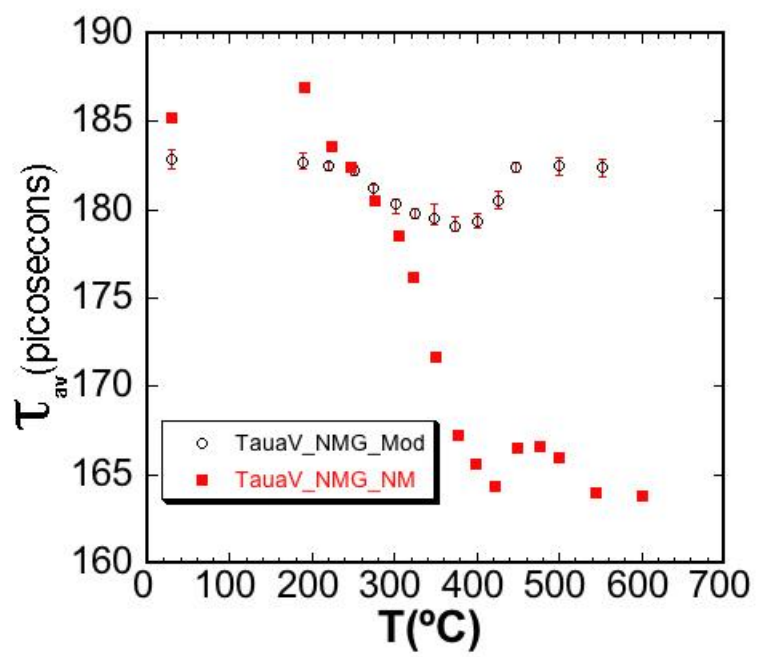

Fig. 4: $\boldsymbol{\tau}_{\mathrm{av}}$ versus isochronal annealing temperature for the NMG_Mod and NMG_NM samples. The error bars for NMG_NM sample are inside the squares.

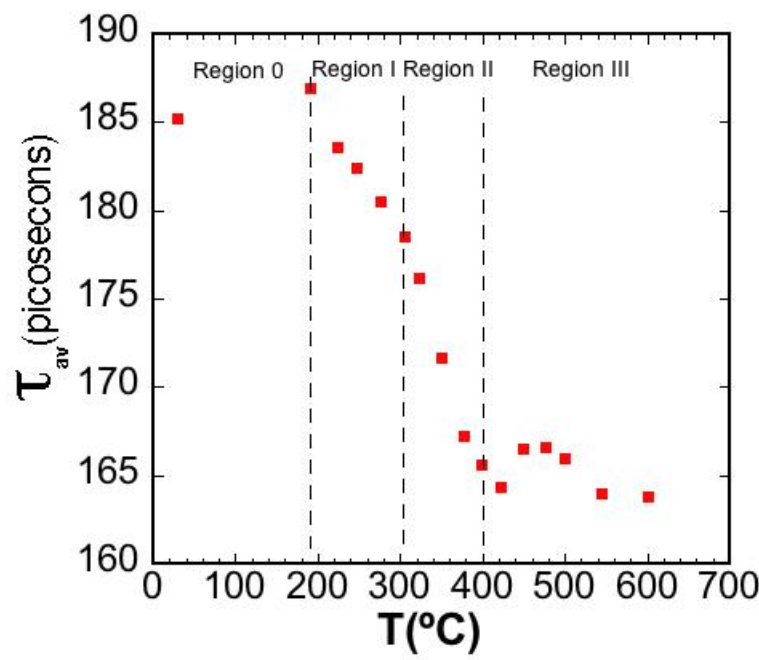

Fig. 5: $\tau_{\mathrm{av}}$ as function of isochronal annealing temperature for NMG_NM. The spectrum is divided in four regions.

Figure 5 shows the behavior of the positron average lifetime as a function of isochronal annealing temperature for NMG_NM sample. The curve has been divided in four regions, denoted by "region 0", "region I", "region II" and "region III". The first average positron lifetime of the curve amounts to $185 \mathrm{ps}$ and corresponds to the sample as-quenched from $900^{\circ} \mathrm{C}$. The second point corresponding to the first annealing at $200^{\circ} \mathrm{C}$ temperature shows a little increase in the average lifetime value. We denote this region "region 0 ". Between $200^{\circ} \mathrm{C}$ and $300^{\circ} \mathrm{C}$ isochronal annealing 
temperature the average lifetime decreases more than $5 \mathrm{ps}$ at a constant rate, of $0.06 \mathrm{ps} /{ }^{\circ} \mathrm{C}$. We have denoted this region as "region I". It has to be indicated that the single exponential fit of the spectra is very good; indeed, it is not possible to decompose the spectra in regions 0 and $\mathrm{I}$. Between $300^{\circ} \mathrm{C}$ and $400^{\circ} \mathrm{C}$ the slope of the positron average lifetime curve increases noticeably. The average lifetime decreases from $179 \mathrm{ps}$ to $165 \mathrm{ps}$ at a constant rate of $0.14 \mathrm{ps} / \mathrm{C}$. We call this region "region II". Even though, one exponential fit of the spectra is very good in region I, the fit is not satisfactory in region II and a second component is necessary to obtain a good fit of the spectra in this region. The behavior found in regions I and II encourages us to think that something different is happening in these two regions. Above $400^{\circ} \mathrm{C}$ the average lifetime remains between $167 \mathrm{ps}$ and $164 \mathrm{ps}$. We call this region "region III".

In the as-quenched NMG_NM sample the positron average lifetime amounts to $185 \mathrm{ps}$ and only one positron lifetime component is present in the spectrum, indicating that all positrons are annihilating from a positron state, which lifetime is $185 \mathrm{ps}$ (or from several positron states with similar positron lifetimes close to $185 \mathrm{ps}$ ). This lifetime value is typical of positrons annihilating from monovacancies in metals [9]. In order, to be certain we have performed self-consistent positron lifetime calculations in $\mathrm{Mn}$, $\mathrm{Ni}$ and $\mathrm{Ga}$ monovacancies of $\mathrm{MnNiGa}$. The calculated positron lifetimes corresponding to $\mathrm{Mn}$ and $\mathrm{Ga}$ monovacancies are 195ps and 196ps; and the one corresponding to Ni monovacancy amounts to $181 \mathrm{ps}$. The calculated values show clearly that the measured lifetime of $185 \mathrm{ps}$ in the as-quenched sample corresponds to positron annihilations in saturation from monovacancies. Figure 5 shows that $\boldsymbol{\tau}_{\mathrm{av}}$ decreases continuously in region I and, as cited previously, it is not possible to decompose the spectra in this region; that is, only one lifetime component is obtained from the spectra in this region. This result indicates that there are two possible explanations for that behavior: 1) the positron state from which positrons are annihilating in saturation (monovacancy) is changing with cycling temperature in region I. 2) positrons are annihilating in saturation from different type of monovacancies and the value of $185 \mathrm{ps}$ is an average of the lifetime of the different monovacancy lifetimes from which positrons annihilate. The first explanation means that in the as-quenched sample only one type of monovacancy is present, and with increasing cycling temperature the monovacancy turns into another type of monovacancy with a shorter positron lifetime. The comparison with the theoretical results indicates clearly that at the end of region I only $\mathrm{V}_{\mathrm{Ni}}$ are present in the sample. The second explanation is in good agreement with the theoretical calculations and takes into account that in the as-quenched sample the three type of monovacancies (or at least the monovacancy with the shorter lifetime and one of the monovacancies with longest ones) are trapping positrons, and with increasing cycling temperature in region I the monovacancies with longest lifetimes, $\mathrm{V}_{\mathrm{Mn}}$ and $\mathrm{V}_{\mathrm{Ga}}$, disappear. At the end of region I only $\mathrm{V}_{\mathrm{Ni}}$ are present in the sample with a concentration higher than the one corresponding to saturation for this technique. Either of the two explanations indicates that at the end of region I $\mathrm{V}_{\mathrm{Ni}}$ is the only monovacancy trapping positrons in the sample.

In region II the positron average lifetime continues decreasing with cycling temperature; however, the lifetime spectra can be decomposed in two components. The long lifetime component obtained in the decomposition is close to $\mathrm{V}_{\mathrm{Ni}}$ positron lifetime, and the short one has values close to 100 ps. This is a clear indication that $\mathrm{V}_{\mathrm{Ni}}$ are not trapping positrons in saturation, and another positron state with shorter lifetime (the bulk of the alloy) is competing in trapping positrons. In other words, $\mathrm{V}_{\mathrm{Ni}}$ are eliminating with increasing isochronal annealing temperature in region II.

Summarizing the positron lifetime measurements performed in the non-modulated sample, NMG_NM, indicate clearly the annihilation of positrons in saturation from monovacancies of the lattice in the as-quenched sample. At cycling temperatures above $200^{\circ} \mathrm{C}$ (region I) there is a recovery state where $\mathrm{V}_{\mathrm{Mn}}$ and $\mathrm{V}_{\mathrm{Ga}}$ disappear and at $300^{\circ} \mathrm{C}$ (end of region I) only $\mathrm{V}_{\mathrm{Ni}}$ are trapping positrons. A new recovery state occurs in region II, where $\mathrm{V}_{\mathrm{Ni}}$ are eliminating.

The behavior of the modulated sample, NMG_Mod, (see figure 4) is very different to the one shown by the non-modulated one analyzed above. It is not possible to decompose the spectra in all the studied temperature range and the positron lifetime value of $182 \mathrm{ps}$ measured below $250^{\circ} \mathrm{C}$ is identical to the value measured above $450^{\circ} \mathrm{C}$. So, positrons are annihilating in saturation from 
monovacancies below $250^{\circ} \mathrm{C}$ and above $450^{\circ} \mathrm{C}$. Therefore, the little valley observed in the temperature range $250-450^{\circ} \mathrm{C}$ can not be understood as an elimination of vacancies. In order to explain this behavior, we have taken into account the temperature at which the martensitic transformation takes place. Figure 2 shows the martensitic transformation temperature changes from $12^{\circ} \mathrm{C}$ for an isochronal annealing temperature of $200^{\circ} \mathrm{C}$ to $28^{\circ} \mathrm{C}$ for an isochronal annealing temperature of $400^{\circ} \mathrm{C}$. Positron annihilation lifetime measurements in figure 4 have been always taken at $22^{\circ} \mathrm{C}$, which was the laboratory stabilized temperature. This means that we have measured the lifetime during the transformation processes. Then we can suppose that the valley in the lifetime spectra of figure 4 of NMG_Mod samples is produced by the coexistence of both martensitic-austenite phases during the transformation. Positrons can be trapped in the interface between both phases.

There exists a clear difference between positron results in the NMG modulated and nonmodulated samples. In non-modulated samples, a clear elimination of vacancies is observed, while in the modulated one, such a decrease of monovacancies is not observed at all. This means that the behavior of vacancies in these two samples is very different.

\section{Comparison between calorimetric and positron lifetime measurements}

As it can be inferred from figures 2 and 3, the temperature range in which Mp changes is the same for both modulated and non-modulated samples, but the position of the exothermic peak is different. For the modulated sample the exothermic peak extends from $250^{\circ} \mathrm{C}$ to $350^{\circ} \mathrm{C}$ and in the non-modulated from $200^{\circ} \mathrm{C}$ to $300^{\circ} \mathrm{C}$. As can be seen in figure 5 the temperature interval in which the positron average lifetime changes is from $200^{\circ} \mathrm{C}$ to $400^{\circ} \mathrm{C}$. The same temperature range in which the exothermic peak appears and $\mathrm{Mp}$ changes. This suggests that the decrease of vacancy concentration in the alloy could be related with the martensite transformation of NMG_NM. Beside, the different position of the two exothermic peaks coincides with region I and region II, so, we can relate these two phenomena. Taken into account that the irreversible process causing the exothermic peak is an ordering process and that ordering requires diffusion of atoms, which can be mediated by vacancies [10], it is logical to assume that vacancies affects the martensitic transformation temperature. Moreover, it is expected that different type of vacancies affect the martensite transformation in a different way.

In the case of the modulated sample, NMG_Mod, the information obtained from the lifetime spectra is that the vacancy concentration is very high in all the annealing temperature range. Indeed, there is saturation positron trapping at defects in the studied temperature range, indicating that the concentration of vacancies has to be higher than the saturation value of the technique for the whole annealing range. This indicates either the vacancy concentration doesn't change or if the vacancy concentration changes it remains above the saturation value. More work has to be done to clarify this point.

\section{Conclusions}

The role of vacancy type defects in the modulated $\left(\mathrm{Ni}_{49.5} \mathrm{Mn}_{28.5} \mathrm{Ga}_{22}\right)$ and non-modulated $\left(\mathrm{Ni}_{52.6}\right.$ $\mathrm{Mn}_{26.7} \mathrm{Ga}_{20.7}$ ) martensite transformations in polycrystalline samples has been studied measuring the effect of thermal treatment on positron lifetime spectra. The spectra have been compared with calorimetric measurements.

The lifetime measurements show a clear difference in the behavior of positron traps (vacancies) in the modulated and non-modulated samples.

In the non-modulated sample, two recovery states, related to elimination of vacancies are observed. It is necessary to take into account at least two types of vacancies to interpret the measurements. The overall vacancy concentration decreases in the same temperature range in which Mp changes. Therefore, it is conclude that vacancies affect the Mp transformation. The way in 
which both are related and the specific mechanism that takes places in the process will be the subject of future investigations.

In the modulated sample we haven't observed any elimination of vacancies. Its behavior is completely different from the NMG_NM. In this sample a greater amount of vacancy type defects exist even, after temperatures above the exothermic peak. This high density of vacancies and the modulation state could be related.

\section{Acknowledgement}

This work has been realized under financial support from the Spanish CICYT and Basque Government under Grants: MAT-2006-12743, MAT-2006-12838 and IT 38207. One of us (D. Mérida) is indebted to the University of the Basque Country (UPV/EHU) for financial support.

\section{References}

[1] K. Ullakko, J. K. Huang, C. Kantner, R. C. O’Handley and V.V Kokorin: Appl. Phys. Lett. Vol. 69 (1996), p. 1966

[2] K.M. Mostafa, N. Van Caenegem, J. de Baerdemaeker, D. Segers and Y. Houbaert: Physica Status Solidi C Vol. 4-10 (2007) p. 3554

[3] I. Hurtado, D. Segers, J. Van Humbeek, L. Dorikens-Vanpraer and C. Dauwe: Scripta Metallurgia \& Materialia Vol. 33-5 (1995), p. 949

[4] A. Dupasquier, G. Kögel, and A. Somoza: Acta Mater. Vol. 52 (2004), p. 4707

[5] J.M. Campillo Robles, E. Ogando and F. Plazaola: J. Phys.: Condens. Matter Vol. 19 (2007), p. 176222

[6] A. T. Zayak, P. Entel, J. Enkovaara, A. Ayuela and R. M. Nieminen: Physical Review B Vol. 68 (2003) p. 132402

[7] V. Sanchez-Alarcos, J. I. Perez-Landazábal, V. Recarte and G. J. Cuello: Acta Materialia Vol. 55 (2007), p. 3883

[8] S. Kustov, J. Pons, E. Cesari and J. Van Humbeeck: Acta Mater. Vol. 52 (2004), p. 3083

[9] J. M. Campillo Robles, F. Plazaola:Defect and Diffusion Forum Vol. 213-215 (2003), p. 141

[10] G. Erdélyi, H. Mehrer, A. W. Imre, T. A. Lograsso and D. L. Schlagel:Intermetallics Vol. 15 (2007), p. 1078 